\title{
Modelagem para o dimensionamento de um sistema de microirrigação utilizando microtubos ramificados ${ }^{1}$
}

\author{
Dinara G. Alves ${ }^{2}$, Marinaldo F. Pinto ${ }^{3}$, Conan A. Salvador ${ }^{3}$, \\ Alexsandro C. S. Almeida ${ }^{3}$, Ceres D. G. C. de Almeida ${ }^{4} \&$ Tarlei A. Botrel $^{3}$
}

RESU M O

Este trabalho foi realizado com o objetivo de se modelar e avaliar o comportamento hidráulico de microtubos ramificados, com base na relação entre vazão e comprimento dos microtubos para uma pressão de entrada, operando sob regime laminar. D ois modelos matemáticos foram estudados, em que um considerou a perda localizada de carga no dimensionamento dos microtubos ramificados e o outro a desconsiderou. $\mathrm{O}$ experimento para validação dos modelos foi conduzido no Laboratório de Hidráulica da Escola Superior de Agricultura "Luiz de Queiroz" (ESALQ -USP), Piracicaba, SP, medindo-se a vazão dos emissores para as pressões de 14,7; 18,6; 39,2 e 58,9 kPa. A configuração do sistema de irrigação consistiu de uma linha lateral com diâmetro nominal (DN) de $10,0 \mathrm{~mm}$, na qual foram inseridos microtubos conectores com DN de $0,8 \mathrm{~mm}$ conectados a um segmento de tubo de derivação e acoplados seis microtubos emissores com $D N$ de $0,7 \mathrm{~mm}$. Verificou-se que, dentre os modelos matemáticos, aquele que considerou a perda localizada de carga apresentou maior exatidão pois teve elevado, um bom índice de Willmott e baixo erro quadrático médio.

Palavras-chave: emissores, tecnologia de irrigação, perda de carga, modelo matemático, baixo custo

\section{Modeling for the design of a micro-irrigation system using branched microtubes}

\begin{abstract}
AB STRACT
This study was carried out with the aim to develop a mathematical model and to evaluate the hydraulic behavior of branched microtubes, based on the relationship between discharge and microtube length for a specific inlet pressure, operating under laminar flow conditions. Two mathematical models were studied in which one considered the local head losses in irrigation system design and the another did not consider. The experiment for model validation was carried out at the Laboratory of Hydraulics, School of Agriculture "Luiz de Q ueiroz" (ESALQ/USP), Piracicaba-SP, where emitter discharges was verified under the pressures of $14.7 ; 18.6 ; 39.2$ and $58.9 \mathrm{kPa}$. The irrigation system configuration consisted of a lateral line with nominal diameter (DN) of $10.0 \mathrm{~mm}$, in which are inserted microtube connectors with $0.8 \mathrm{~mm}$ DN . These connectors are connected to a derivation tube segment, where six emitter microtubes with 0.7 $\mathrm{mm}$ DN were attached. It was verified that the model which considered the local head losses showed better accuracy than other mathematical models, as this model showed a good Willmott index and a low mean quadratic error.
\end{abstract}

Key words: emitter, irrigation technology, head loss, mathematical model, low cost

\footnotetext{
${ }^{1}$ Parte da Dissertação de Mestrado do primeiro autor, apresentada à ESALQ/U SP, Piracicaba, SP

2 D outorando em Eng. de Sistemas Agrícolas, ESALQ/U SP. Av. Pádua Dias, 11, CEP 13418-900. Piracicaba, SP. Fone: (19) 3447-8549. E-mail: dinara@usp.br

3 ESALQ/U SP. E-mail: marinald@esalq.usp.br; conan@usp.br; acdsalme@esalq.usp.br; tabotrel@esalq.usp.br

${ }^{4}$ CODAI/U FRPE, Rua Doutor Francisco Correia, 643, Centro, CEP 54735-000, São Lourenço da Mata, PE. E-mail: ceres@codai.ufrpe.br
} 


\section{INTRODUÇÃO}

O microtubo é um tipo de gotejador feito de polietileno, com diâmetros entre 0,6 e 1,5 mm e já foi muito utilizado no mundo todo, no início da microirrigação (gotejamento). No entanto, sua alta sensibilidade à variação de temperatura e pressão e susceptibilidade ao entupimento, foram fatores preponderantes para seu desuso ao longo do tempo. Em consequência, emissores menos exigentes em mão-de-obra na instalação, mais resistentes a danos mecânicos e menos sensíveis às variações de pressão e temperatura se tornaram predominantes no mercado.

Todavia, alguns fatores têm contribuído para a retomada da adoção da tecnologia de microtubos, sobretudo a melhoria dos sistemas de filtragem. A evolução da tecnologia de filtragem de água nas últimas décadas tem viabilizado a utilização de sistemas mais sensíveis à obstrução. No entanto, os microtubos são indicados para diversas situações, inclusive para locais onde existem grandes diferenças de pressão devido a desníveis de topografia e a grandes perdas de carga, pois é possível compensar a variação de pressão variando o comprimento do microtubo, e consequentemente, obter vazão uniforme ao longo da linha lateral. Salienta-se que emissores autocompensantes também são indicados para esta situação visto que possuem um dispositivo que compensa a variabilidade da pressão, proporcionando uniformização da vazão no campo apesar de seu elevado custo quando comparado com os microtubos emissores. Experimento realizado por Souza et al. (2006) no qual utilizaram sistema de irrigação por gotejamento com microtubos, os autores obtiveram uniformidade de aplicação de água de $97,0 \%$, considerado valor excelente e ainda compararam o custo dos microtubos com o dos tubos-gotejadores e verificaram que a utilização de linhas laterais com microtubos proporcionou uma economia de $54,4 \%$.

Ressalta-se que a utilização do sistema de irrigação por gotejamento com microtubos deve ser realizada em pequenas áreas visto que a montagem deste sistema é trabalhosa e requer muita mão-de-obra. Neste sentido, a tecnologia de irrigação por microtubos se apresenta como alternativa viável para pequenos produtores, podendo conferir redução de custo de produção em virtude do baixo preço de aquisição do sistema (Souza et al., 2006; 2009). A maioria desses produtores trabalha com agricultura familiar, a qual é responsável por garantir boa parte da segurança alimentar, situando-se como importante fornecedora de alimentos para o mercado interno brasileiro (IBGE, 2006). Em geral, esses agricultores são de baixa renda e, por consequência, apresentam baixa capacidade de adoção de tecnologias em irrigação.

Em contrapartida, para que o sistema de irrigação com microtubos seja dimensionado corretamente e, em consequência, possibilite que o pequeno agricultor obtenha melhoria na produtividade e nolucro, necessita-se de um projeto criterioso e detalhado. Deste modo, Pizarro Cabello (1996) recomenda que as características desses emissores sejam determinadas experimentalmente em laboratório, utilizando-se um modelo adequado de representação da perda de carga, conforme teoria hidráulica; contudo, a determinação do diâmetro real do microtubo é outro fator que deve ser considerado, uma vez que qualquer erro na sua atribuição poderá ocasionar grande diferença na estimativa da vazão.

Neste sentido, Souza \& Botrel (2004) e Souza et al. (2011) desenvolveram modelos matemáticos para dimensionamento de emissores microtubos, apresentando coeficiente de determinação de 99,67 e exatidão de 97,0\%, respectivamente, porém não existem modelos para o dimensionamento de microtubos ramificados, por se tratar de uma configuração inovadora. O sistema é caracterizado por possuir menor custo em comparação com os sistemas atuais, que utilizam microtubos, tendo em vista a menor quantidade de material necessário à sua implantação.

Ante o exposto, este trabalho objetivou modelar e avaliar o comportamento hidráulico de microtubos ramificados com base na relação entre vazão e comprimento dos microtubos para determinada pressão de entrada, operando sob regime laminar.

\section{Material e MÉTODOS}

A condução deste trabalho foi realizada no Laboratório de Hidráulica do Departamento de Engenharia de Biossistemas da Escola Superior de Agricultura "Luiz de Queiroz"/USP, situado no município de Piracicaba, SP.

\section{Diâmetro interno dos microtubos}

Avaliaram-se microtubos de cor verde e vermelha, com diâmetros nominais de 0,7 e $0,8 \mathrm{~mm}$, respectivamente com comprimento de $15,0 \mathrm{~m}$ cada um. Os referidos microtubos foram submetidos a uma pressão constante de $12,4 \mathrm{kPa}$ estimada com base na distância entre o nível da água do reservatório superior e o final do microtubo. A determinação do diâmetro interno do microtubo foi realizada através de medições hidrodinâmicas em laboratório, conforme metodologia proposta por Almeida \& Botrel (2010). Foram realizadas, também, três repetições em cada ensaio.

Os diâmetros internos dos microtubos foram calculados por meio de dados obtidos em ensaio sob regime laminar, aplicados na equação proposta por Almeida \& Botrel (2010) Eq. 1.

$$
\mathrm{D}=\left(\frac{0,08262647 \mathrm{Q}^{2}+4,153269 \mathrm{LvQ}}{\mathrm{Z}}\right)^{0,25}
$$

em que:

D - diâmetro interno do microtubo, $\mathrm{m}$

Q - vazão no microtubo, $\mathrm{m}^{3} \mathrm{~s}^{-1}$

$v$ - viscosidade cinemática, $\mathrm{m}^{2} \mathrm{~s}^{-1}$

$\mathrm{Z}$ - carga potencial, $\mathrm{m}$

L - comprimento do microtubo, $\mathrm{m}$

\section{Comprimento dos microtubos}

A configuração do sistema de irrigação consiste de uma linha lateral com diâmetro nominal (DN) de 10 mm e nela são inseridos microtubos conectores com $\mathrm{DN}$ de $0,8 \mathrm{~mm}$ conectados a um segmento de tubo de derivação, além de acoplados seis microtubos emissores com DN de 0,7 mm (Figura 1). 


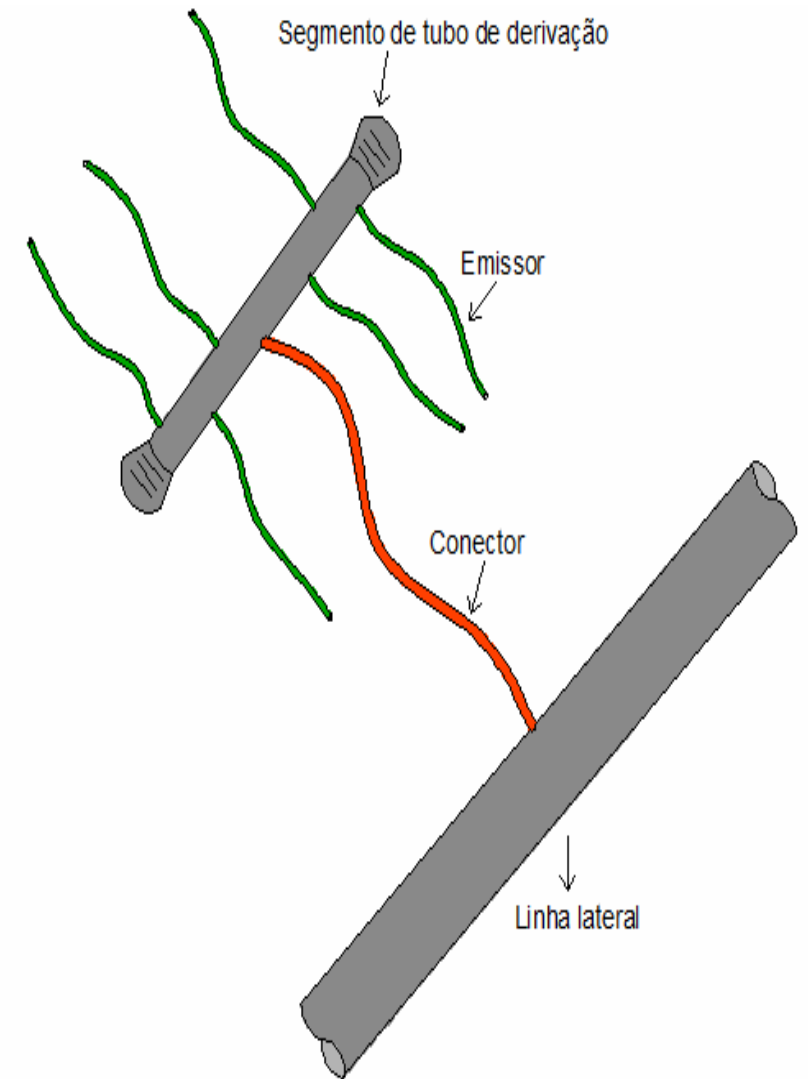

Figura 1. Esquema do sistema de irrigação utilizando-se microtubos ramificados

No desenvolvimento do modelo para o dimensionamento do emissor proposto, assumiu-se que os diâmetros internos dos microtubos foram aqueles determinados hidrodinamicamente em laboratório, aumentando a precisão dos cálculos. Neste experimento foram utilizados microtubos de polietileno, com diâmetro nominal de $0,7 \mathrm{~mm}$ para microtubos emissores e 0,8 mm para microtubos conectores, cujo diâmetro real dos microtubos foi de 0,695 e $0,887 \mathrm{~mm}$, respectivamente.

O cálculo do comprimento dos conectores e emissores para a validação do modelo foi feito com base em uma planilha eletrônica, cujas variáveis independentes foram: pressão, diâmetro e vazão. Para calcular o comprimento dos microtubos foram admitidos os seguintes valores inicialmente propostos no projeto: vazão de $0,2 \mathrm{~L} \mathrm{~h}^{-1}$ para o microtubo emissor e de $1,2 \mathrm{~L} \mathrm{~h}^{-1}$ para microtubo conector; viscosidade de $1,01 \times 10^{-6}$ $\mathrm{m}^{2} \mathrm{~s}^{-1}$ para a temperatura de $20^{\circ} \mathrm{C}$; pressão de alimentação de $14,7 \mathrm{kPa}$ e pressão no segmento de tubo de derivação de 2,94 $\mathrm{kPa}$.

O fator de atrito (f) e o número de Reynolds (NR) foram calculados por meio das Eqs. 2 e 3, respectivamente; ressaltase a utilização da Eq. 2 pelo fato do regime de escoamento ser laminar (NR $\leq 2000)$. Para o cálculo da perda de carga unitária foi utilizada a Eq. 4. No cálculo da perda de carga no microtubo conector e emissor, utilizou-se a equação de Bernoulli (Eq. 5). Calculou-se o comprimento do microtubo conector e emissor dividindo-se a perda de carga (hf) pela perda de carga unitária (J) do microtubo.

$$
\begin{gathered}
\mathrm{NR}=\frac{\mathrm{VD}}{v} \\
\mathrm{~J}=\mathrm{f} \frac{\mathrm{V}^{2}}{2 \mathrm{gD}} \\
\mathrm{hf}=\frac{\mathrm{P}_{2}-\mathrm{P}_{1}}{\gamma}+\frac{\mathrm{V}_{2}^{2}-\mathrm{V}_{1}^{2}}{2 \mathrm{~g}}+\mathrm{Z}_{2}-\mathrm{Z}_{1}
\end{gathered}
$$

em que:

$$
\begin{aligned}
& \mathrm{V} \text { - velocidade da água no microtubo, } \mathrm{m} \mathrm{s}^{-1} \\
& \mathrm{f} \text { - fator de atrito da fórmula universal, adimensional } \\
& \mathrm{g} \quad \text { - aceleração da gravidade, } \mathrm{m} \mathrm{s}^{-2} \\
& \mathrm{~J}
\end{aligned}
$$

A pressão no segmento de tubo de derivação foi calculada por meio da Eq. 6 .

$$
\mathrm{Pst}_{\text {calc }}=\mathrm{P}_{\mathrm{e}}-\mathrm{hf}_{\mathrm{c}}
$$

em que:

$\mathrm{Pst}_{\text {calc }}$ - pressão no segmento de tubo de derivação calculada, m.c.a

$\mathrm{P}_{\mathrm{e}}$ - pressão na entrada do microtubo conector, m.c.a

$\mathrm{hf}_{\mathrm{c}}$ - perda de carga no microtubo conector, m.c.a.

Realizaram-se ensaios em laboratório com o sistema de irrigação funcionando sob pressões de 14,7; 18,6; 39,2 e 58,9 $\mathrm{kPa}$, para verificar se a pressão no segmento de tubo de derivação calculada estava de acordo com a pressão no segmento de tubo de derivação, observada experimentalmente.

\section{Perda localizada de carga}

Com base nos ensaios realizados em laboratório observouse a existência de perda localizada de carga, a qual precisou ser analisada para verificar sua importância no dimensionamento do microtubo emissor visto que a mesma só pode ser determinada empiricamente, haja vista que, comumente, os diâmetros dos microtubos fornecidos pelo fabricante não são iguais ao diâmetro real.

Para o cálculo da perda localizada de carga (Hloc) utilizouse a Eq. 7; em seguida, determinou-se o coeficiente de perda localizada de carga (K) Eq. 8 .

$$
\begin{gathered}
\text { Hloc }=\text { Pst }_{\text {calc }}-\text { Pst }_{\mathrm{obs}} \\
\mathrm{K}=\text { Hloc } \frac{2 \mathrm{~g}}{\mathrm{~V}^{2}}
\end{gathered}
$$

em que:

$\mathrm{Pst}_{\mathrm{obs}}$ - pressão no segmento de tubo de derivação observada, m.c.a. 
Após a determinação de K realizou-se o ajuste no comprimento do microtubo conector para a pressão de alimentação de $14,7 \mathrm{kPa}$.

Considerando que o seguimento de tubo de derivação e os microtubos não apresentam uniformidade construtiva, fica evidente que a caracterização do emissor por meio do coeficiente de perda localizada de carga, é um fator complicador para aplicação prática do modelo de dimensionamento de microtubos ramificados.

Com base no exposto, elaboraram-se dois modelos matemáticos desconsiderando-se, em um, a perda localizada de carga (modelo I) e, no outro, considerando-a (modelo II). Posteriormente, o desempenho dos dois modelos foi avaliado mediante medição da vazão nos gotejadores. Ressalta-se que o modelo I é mais simples e não necessita de resultados empíricos para sua determinação, ao contrário do modelo II.

Os modelos utilizados no cálculo do comprimento do microtubo conector para sua utilização ao longo da linha lateral no sistema de irrigação, estão descritos nas Eqs. 9 e 10.

$$
\begin{gathered}
\mathrm{L}_{\mathrm{c}}=\left(\frac{\mathrm{P}_{\mathrm{ei}}-\mathrm{Pst}}{\mathrm{J}}\right) 100 \\
\mathrm{~L}_{\mathrm{c}}=\left(\frac{\mathrm{P}_{\mathrm{ei}}-\mathrm{Pst}-\mathrm{Hloc}}{\mathrm{J}}\right) 100
\end{gathered}
$$

A pressão na entrada do microtubo conector no ponto "i" $\left(\mathrm{P}_{\mathrm{ei}}\right)$ foi determinada por meio da Eq. 11.

$$
\mathrm{P}_{\mathrm{ei}}=\mathrm{P}_{\mathrm{ei}-1}+\mathrm{hf}_{\mathrm{i}-1} \pm \mathrm{IE}_{\mathrm{c}}
$$

em que:

$\mathrm{L}_{\mathrm{c}}$ - comprimento do microtubo conector, $\mathrm{cm}$

$\mathrm{i}^{\mathrm{c}}$ - posição do microtubo conector ao longo da linha lateral

$\mathrm{P}_{\text {ei-1 }}$ - pressão no ponto anterior ao ponto "i", m.c.a.

$\mathrm{hf}_{\mathrm{i}-1}$ - perda de carga no trecho "i-1", m.c.a.

I - declividade no terreno, decimal

$\mathrm{E}_{\mathrm{c}}$ - espaçamento entre microtubos conectores, $\mathrm{m}$.

\section{Vazão nos emissores}

Para realização do cálculo da vazão nos emissores consideraram-se a perda de carga no microtubo conector, a perda de carga do microtubo emissor e a pressão na entrada do microtubo conector (ponto de inserção do microtubo na linha lateral).

A pressão na entrada do microtubo conector foi determinada através das Eq. 12 e 13:

$$
\begin{gathered}
\mathrm{P}_{\mathrm{e}}=\mathrm{hf}_{\mathrm{c}}+\mathrm{hf}_{\mathrm{e}} \\
\mathrm{P}_{\mathrm{e}}=\mathrm{hf}_{\mathrm{c}}+\mathrm{hf}_{\mathrm{e}}+\text { Hloc }
\end{gathered}
$$

em que:

$\mathrm{hf}_{\mathrm{e}}$ - perda de carga no microtubo emissor, m.c.a.
A vazão no microtubo conector é igual ao somatório das vazões nos microtubos emissores Eq. 14.

$$
\mathrm{Q}_{\mathrm{c}}=\mathrm{nQ}_{\mathrm{e}}
$$

em que:

n - número de microtubos emissores, adimensional

$\mathrm{Q}_{\mathrm{e}}$ - vazão no microtubo emissor, $\mathrm{m}^{3} \mathrm{~s}^{-1}$

$\mathrm{Q}_{\mathrm{c}}$ - vazão no microtubo conector, $\mathrm{m}^{3} \mathrm{~s}^{-1}$

Substituindo a equação de Darcy-Weisbach Eq. 15 nas Eqs. 12 e 13 e se realizando as devidas simplificações, determinaramse as Eqs. 16 e 17, respectivamente:

$$
h f=\frac{8 f L Q^{2}}{g \pi^{2} D^{5}}
$$

$$
\mathrm{Q}_{\mathrm{e}}=\frac{-\frac{128 v}{\pi \mathrm{g}}\left(\frac{\mathrm{nL}}{\mathrm{D}_{\mathrm{c}}^{4}}+\frac{\mathrm{L}_{\mathrm{e}}}{\mathrm{D}_{\mathrm{e}}^{4}}\right)+\sqrt{\left(\frac{128 v}{\pi \mathrm{g}}\left(\frac{\mathrm{nL}}{\mathrm{D}_{\mathrm{c}}^{4}}+\frac{\mathrm{L}_{\mathrm{e}}}{\mathrm{D}_{\mathrm{e}}^{4}}\right)\right)^{2}-4\left(\frac{8 \mathrm{~K}_{\mathrm{c}} \mathrm{n}^{2}}{\pi^{2} \mathrm{gD}_{\mathrm{c}}^{4}} \times-\mathrm{P}_{\mathrm{e}}\right)}}{\frac{16 \mathrm{~K}_{\mathrm{c}} \mathrm{n}^{2}}{\pi^{2} \mathrm{gD}_{\mathrm{c}}^{4}}}(17)
$$

em que:
$\mathrm{D}_{\mathrm{c}}$ - diâmetro do microtubo conector, $\mathrm{m}$
$\mathrm{D}_{\mathrm{e}}$ - diâmetro do microtubo emissor, $\mathrm{m}$
$\mathrm{L}_{\mathrm{c}}$ - comprimento do microtubo conector, $\mathrm{m}$
$\mathrm{L}_{\mathrm{e}}$ - comprimento do microtubo emissor, $\mathrm{m}$

A vazão observada em laboratório foi comparada com as vazões calculadas por meio das Eq. 16 e 17, para avaliação dos modelos desenvolvidos.

\section{Validação do modelo}

Montaram-se duas bancadas de teste, a primeira visando funcionar a uma pressão de $14,7 \mathrm{kPa}$, composta de uma linha lateral com diâmetro interno de $13 \mathrm{~mm}$, três microtubos conectores dos quais derivaram os microtubos emissores, um filtro de disco de 120 mesh, um reservatório para abastecimento d'água, um piezômetro, dezoito coletores de massa conhecida para a coleta de água e dois registros, um para controlar a entrada de água no reservatório e o outro para controlar a entrada de água nos emissores (Figura 2).

A água proveniente de uma caixa d'água passava pelo filtro de disco e por um registro agulha para depois abastecer o reservatório, o qual possuía um dreno utilizado para eliminar o excesso de água recebida da caixa d'água, mantendo o nível constante. O piezômetro instalado no início da tubulação foi utilizado para monitorar a pressão de alimentação da lateral. 


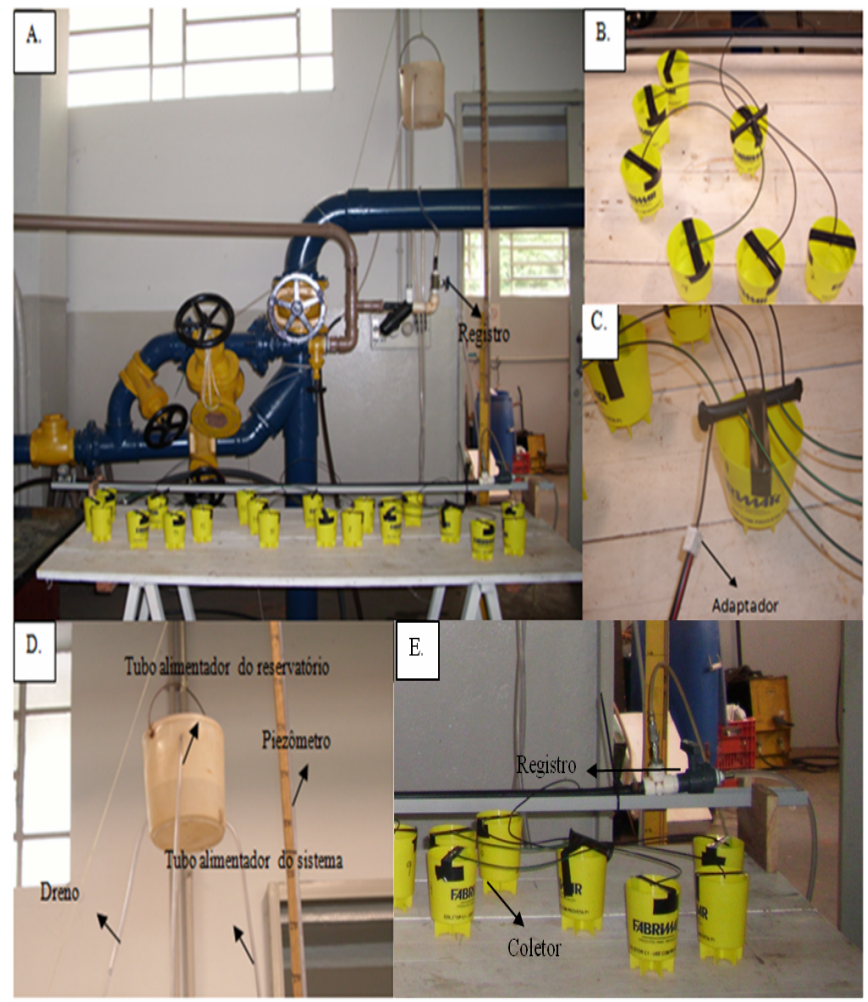

Figura 2. Bancada de testes para determinação da vazão dos microtubos sob pressão de $14,72 \mathrm{kPa}(\mathrm{A})$, emissor com derivação $(B)$, adaptador utilizado para conectar 0 microtubo ao piezômetro $(C)$, reservatório alimentador do sistema (D), coletor para coleta de vazão (E)

A distância entre conexões dos microtubos conectores foi de aproximadamente $25 \mathrm{~cm}$, visando evitar a influência de causas perturbadoras no escoamento. Durante os ensaios os três microtubos conectores foram colocados simultaneamente em funcionamento; enfim, a água foi coletada durante $60 \mathrm{~min}$.

Na segunda bancada de ensaios o abastecimento de água para os emissores ocorreu por meio de uma caixa d'água, elevada a uma altura de $15 \mathrm{~m}$, conferindo uma pressão de 147,2 kPa no início da linha lateral. Os microtubos utilizados na primeira bancada de testes foram novamente utilizados na segunda bancada sendo desta vez ensaiados sob pressões de 18,6; 39,2 e 58,9 $\mathrm{kPa}$. A regulagem das pressões se deu por meio de um registro e as mesmas foram determinadas por meio de um manômetro digital instalado no início da tubulação. É importante salientar que não foi possível utilizar a pressão de $14,7 \mathrm{kPa}$ nesta configuração em virtude da elevada instabilidade apresentada no momento da leitura no manômetro para pressões inferiores a $18,6 \mathrm{kPa}$. A água foi coletada durante 50 , 45 e 30 min para as pressões de 18,$6 ; 39,2$ e 58,9 kPa, respectivamente.

Utilizaram-se os ensaios realizados nas primeira e segunda bancadas, para observar a pressão no segmento de tubo de derivação $\left(\right.$ Pst $\left._{\text {obs }}\right)$. Para esta verificação realizou-se um furo no segmento de tubo de derivação no qual foi introduzido um microtubo que se ligou, por meio de um adaptador, a um tubo de diâmetro maior (Figura 2C). Este último foi conectado a um piezômetro utilizado no monitoramento da pressão no segmento de tubo de derivação. A leitura da carga de pressão foi realizada três vezes para cada condição de ensaio.

Em ambos os ensaios obteve-se o valor da vazão pela média de três repetições, para cada pressão avaliada. Após cada coleta os coletores com água foram mensurados em balança de precisão de $0,01 \mathrm{~g}$, e a massa transformada em volume ao dividi-la pela massa específica da água. A temperatura da água foi monitorada a fim de se obter os valores da viscosidade cinemática e o valor do seu peso específico. As vazões foram determinadas mediante a razão entre o volume e o tempo de cada coleta.

Realizou-se a comparação entre os valores de vazões observadas $\left(\mathrm{Q}_{\mathrm{eo}}\right)$ e calculadas $\left(\mathrm{Q}_{\mathrm{ec}}\right)$ aplicando-se o erro quadrático médio (EQM) Eq. 18 e o coeficiente de correlação de Willmott et al. (1985) Eq. 19.

$$
\begin{gathered}
\mathrm{EQM}=\sqrt{\frac{\sum_{\mathrm{i}=1}^{\mathrm{N}}\left(\mathrm{Q}_{\mathrm{eo}}-\mathrm{Q}_{\mathrm{ec}}\right)^{2}}{\mathrm{~N}}} \\
\mathrm{~d}=1-\frac{\sum_{\mathrm{i}=1}^{\mathrm{N}}\left(\mathrm{Q}_{\mathrm{ec}}-\mathrm{Q}_{\mathrm{eo}}\right)^{2}}{\sum_{\mathrm{i}=1}^{\mathrm{N}}\left(\left|\mathrm{Q}_{\mathrm{ec}}-\overline{\mathrm{Q}_{\mathrm{eo}}}\right|+\mid \mathrm{Q}_{\mathrm{eo}}-\overline{\mathrm{Q}_{\mathrm{eo}}}\right)^{2}}
\end{gathered}
$$

em que:

d - índice de concordância de Willmott, \%

$\mathrm{Q}_{\mathrm{eo}}$ - vazão do emissor observada, $\mathrm{m}^{3} \mathrm{~s}^{-1}$

$\mathrm{Q}_{\mathrm{ec}}$ - vazão do emissor calculada, $\mathrm{m}^{3} \mathrm{~s}^{-1}$

$\mathrm{N}$ - número total de observações.

\section{RESULTADOS E DISCUSSÃO}

\section{Comprimento dos microtubos}

Os resultados da planilha eletrônica para o cálculo do comprimento do microtubo emissor e do conecto,r podem ser visualizados na Tabela 1. Esses dados são a base dos ensaios realizados em laboratório, visando obter a relação entre pressão e vazão.

O comprimento do microtubo conector foi calculado para testes em laboratório pois, em campo, seu comprimento é variável devido às perdas de carga que ocorrem ao longo da linha lateral. Esta variação de comprimento visa compensar a perda de carga existente na linha lateral e no microtubo conector mantendo uniforme, assim, a vazão nos microtubos emissores (Souza et al., 2006; Almeida et al., 2009).

Para que a vazão determinada em laboratório fosse a mais próxima possível de $0,2 \mathrm{~L} \mathrm{~h}^{-1}$, foi necessário reduzir o comprimento do microtubo devido à perda localizada de carga. Por conseguinte, o comprimento deste microtubo conector, que antes era de 0,530 , passou a ser de $0,387 \mathrm{~m}$, ou seja, foi necessário realizar a redução no comprimento do microtubo em $27,0 \%$. 
Tabela 1. Parâmetros utilizados para determinação do comprimento do microtubo emissor e conector

\begin{tabular}{|c|c|c|c|c|c|c|c|c|c|c|c|c|c|c|c|}
\hline \multicolumn{8}{|c|}{ Microtubo emissor } & \multicolumn{8}{|c|}{ Microtubo conector } \\
\hline \multirow{2}{*}{$\begin{array}{l}\text { Vazão } \\
\left(L ~^{-1}\right)\end{array}$} & \multirow{2}{*}{$\underset{(\mathrm{mm})}{D}$} & \multirow{2}{*}{ NR } & \multirow{2}{*}{ f } & \multirow{2}{*}{$\underset{\left(\mathrm{m} \mathrm{m}^{-1}\right)}{J}$} & \multirow{2}{*}{$\begin{array}{l}\text { Pst* } \\
\text { (kPa) }\end{array}$} & hf & $\mathbf{L}$ & \multirow{2}{*}{$\begin{array}{l}\text { Vazão } \\
\left(L^{-1}\right)^{-1}\end{array}$} & \multirow{2}{*}{$\underset{(\mathrm{mm})}{D}$} & \multirow{2}{*}{ NR } & \multirow{2}{*}{$f$} & \multirow{2}{*}{$\underset{\left(m^{-1}\right)}{J}$} & \multirow{2}{*}{$\begin{array}{l}\mathbf{P}_{\mathrm{e}}^{*} \\
(\mathrm{kPa})\end{array}$} & hf & $\mathbf{L}$ \\
\hline & & & & & & & & & & & & & & \multicolumn{2}{|c|}{ (m) } \\
\hline 0,2 & 0,695 & 100,7 & 0,64 & 0,99 & 2,94 & 0,30 & 0,300 & 1,2 & 0,887 & 473,7 & 0,14 & 2,26 & 14,70 & 1,20 & 0,530 \\
\hline
\end{tabular}

D - diâmetro interno do microtubo; NR - número de Reynolds; $\mathrm{f}$ - fator de atrito; I - perda de carga unitária; Pst: - pressão no segmento de tubo de derivação; hf - perda de carga; $L$ - comprimento do microtubo; $P_{e}$ - pressão na entrada do microtubo conector

* Valores predefinidos

\section{Avaliação hidráulica do sistema}

As Figuras 3, 4, 5 e 6, que serão mostradas a seguir, se referem aos testes realizados sob pressões de alimentação de 14,$7 ; 18,6 ; 39,2$ e $58,9 \mathrm{kPa}$, com emissores dimensionados desprezando a perda localizada de carga. Segundo Frizzone et al. (1998) a relação entre vazão e pressão, a perda de carga ao longo da linha lateral e no emissor, constituem as características hidráulicas que influenciam diretamente o desempenho do sistema.

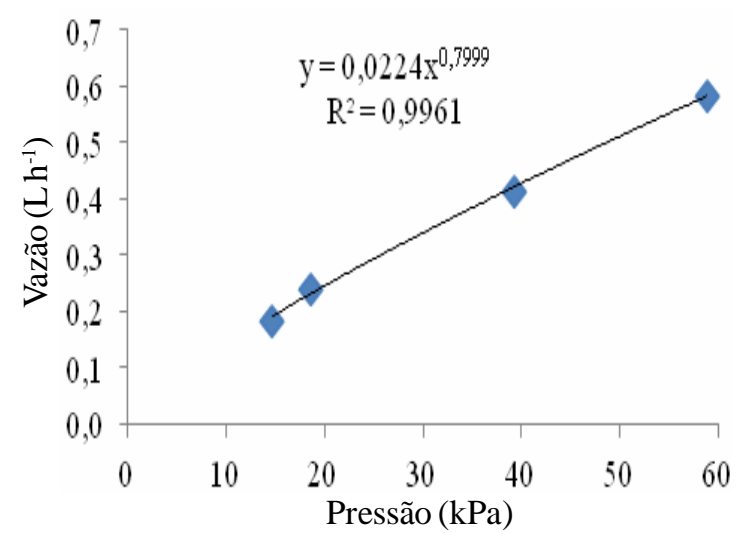

Figura 3. Curva vazão versus pressão do sistema de irrigação com derivação

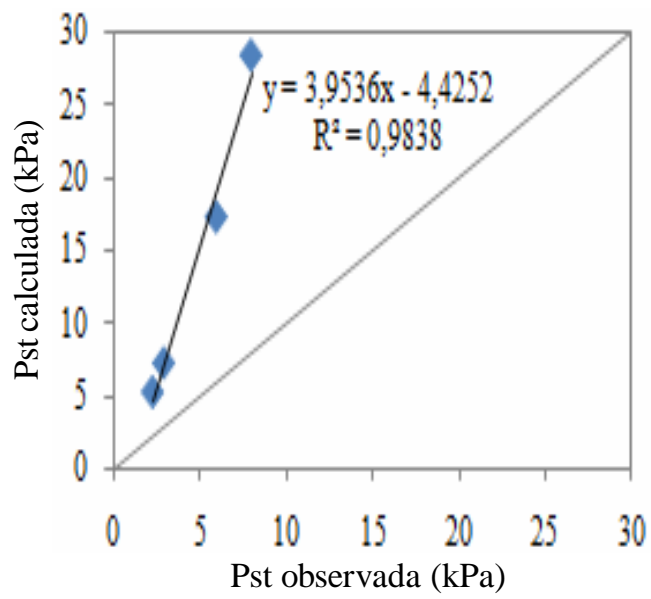

Figura 4. Relação entre pressão no segmento de tubo de derivação (Pst) calculada e observada

A curva da relação entre a vazão e pressão dos microtubos pode ser visualizada na Figura 3. Karmeli (1977) comenta que emissores de longo percurso podem ter expoente de vazão variando de 0,5 a 1,0; portanto, o expoente visualizado na Figura 3 , cujo valor é 0,7999 , está coerente com o que preconiza o autor.

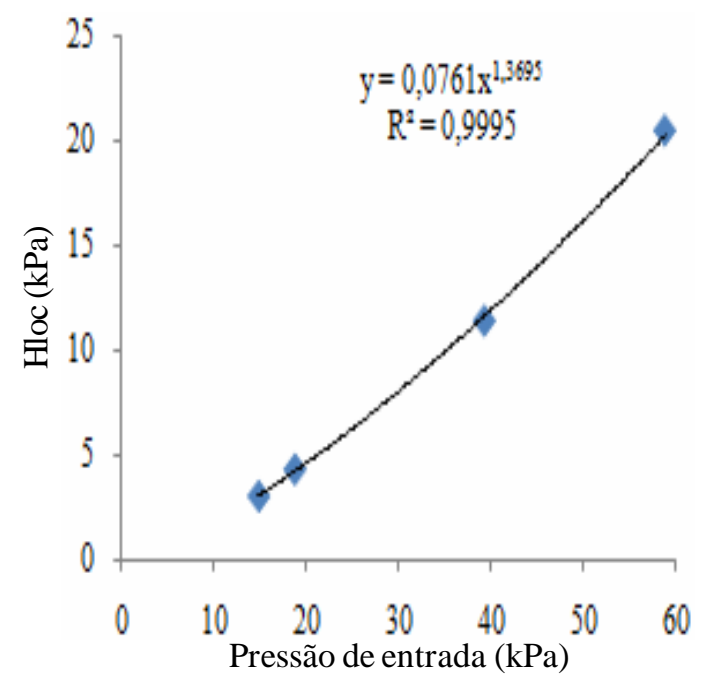

Figura 5. Relação entre perda localizada de carga (HIoc) e pressão de entrada

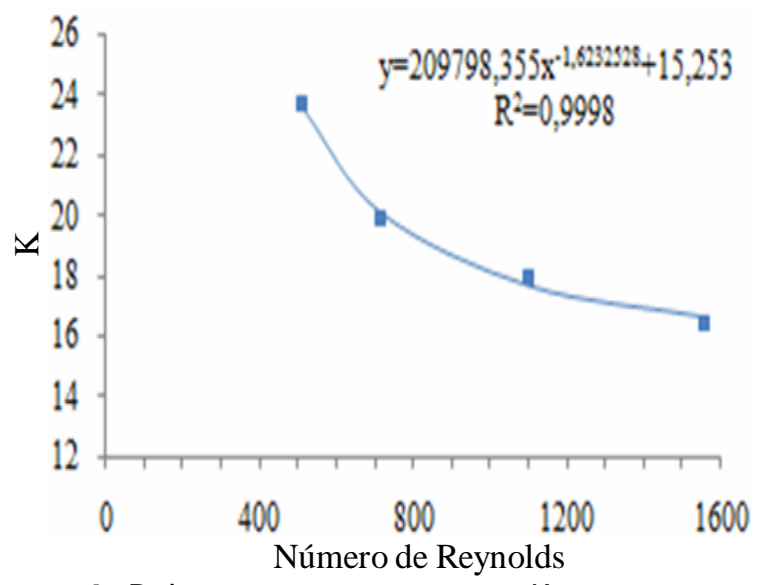

Figura 6. Relação entre a constante $\mathrm{K}$ e o número de Reynolds

A relação entre a pressão no segmento de tubo de derivação (Pst) calculada com a observada, pode ser visualizada na Figura 4. As Pst observadas foram de 2,3; 2,9; 6,0 e 8,0 kPa e diferiram das Pst determinadas nos cálculos, que foram de 5,3; 7,3; 17,3 e 28,4 kPa, apresentando valores inferiores aos estimados para as pressões de alimentação utilizadas nos ensaios, constatando-se que estava ocorrendo perda localizada de carga, isto ocorre quando o fluido sofre alguma perturbação brusca em seu escoamento, por algum elemento nele inserido, causando turbulência local (Cardoso \& Frizzone, 2007; Zitterell et al., 2009; Gomes et al., 2010).

Segundo Azevedo Netto (1998), as perdas localizadas podem ser desprezadas nas tubulações longas cujo comprimento 
exceda cerca de 40000 vezes o diâmetro. São ainda desprezíveis nas canalizações em que a velocidade é baixa e o número de peças especiais não é grande. No entanto, isto não se enquadra neste caso uma vez que o comprimento do microtubo conector é pequeno e, além disso, no segmento de tubo derivam seis emissores. O microtubo conector ocasionou perdas localizadas de carga decorrentes da inserção de parte de seu corpo no interior da linha lateral e no segmento de tubo de derivação, funcionando como ponto de formação, ou acréscimo, de turbulência. Além disso, as mudanças na direção do fluxo d'água contribuíram para essas perdas.

A medida em que a pressão de entrada aumentava a perda localizada de carga também foi ampliada, ocorrência devida ao aumento da velocidade de escoamento, elevando a Hloc, evento verificado também no trabalho experimental realizado por Gomes et al. (2010). Para as pressões de 14,7; 18,6; 39,2 e 58,9 kPa, as perdas localizadas de carga foram de 3,$0 ; 4,3 ; 11,3$ e $20,4 \mathrm{kPa}$ respectivamente, ou seja, essas perdas localizadas ocorridas pelo microtubo conector foram elevadas e devem ser consideradas no dimensionamento hidráulico do microtubo ramificado (Figura 5).

Segundo Porto (2006), o K é um coeficiente adimensional que depende da geometria da conexão, do número de Reynolds, da rugosidade da parede e, em alguns casos, das condições do escoamento, como a distribuição da vazão em ramificação.

Na Figura 6 observou-se que o valor de K, que foi de 23,7; 19,2; 18,0 e 16,5, variou para cada condição ensaiada, reduzindo com o aumento do número de Reynolds, de 511,7; 717,6; 1102,6 e 1155,3 respectivamente, corroborando com os resultados observados por Rettore Neto et. al. (2009). De acordo com Souza \& Botrel (2004), o K não é um valor constante em situações de baixo NR e, sim, uma função do NR. Como resultado, pode-se considerar que o efeito das forças viscosas altera sensivelmente o valor de $\mathrm{K}$ quando se trabalha com número de Reynolds sob regime laminar.

Os valores de vazão tiveram índice de concordância de Willmott de $81,61 \%$ para o modelo I (sem Hloc) e de 99,99\% para o modelo II (com Hloc). O erro quadrático médio (EQM) apresentou valores de 0,156 (modelo I) e $0,003 \mathrm{~L} \mathrm{~h}^{-1}$ (modelo II), ou seja, os valores de vazão medidos do modelo II ficaram próximos do valor de vazão de projeto $\left(0,2 \mathrm{~L} \mathrm{~h}^{-1}\right)$, o que demonstra a qualidade do modelo matemático utilizado no dimensionamento dos microtubos. Apesar do modelo I ter um índice de concordância de Willmott aparentemente elevado, o valor obtido não pode ser considerado satisfatório tendo em vista que a vazão nos emissores é fator primordial para a uniformidade e eficiência do sistema, sendo inaceitável desvio superior a 7,0\% segundo a norma da ABNT/ISO 9261, ABNT (2006). Portanto, os valores de índice de concordância de Willmott (d) e EQM demonstraram que o modelo II teve melhor desempenho (Tabela 2). Resultados semelhantes a esses foram verificados no trabalho de Souza \& Botrel (2004) que desenvolveram modelos matemáticos para dimensionamento de sistemas de irrigação, utilizando microtubos.

O melhor ajuste do modelo II comprova que é pertinente considerar a perda localizada de carga no dimensionamento dos microtubos ramificados funcionando sob regime laminar,
Tabela 2. Índice de concordância de Willmott (d) e erro quadrático médio (EQ M) para o modelo I e modelo II

\begin{tabular}{cccc}
\hline & Modelo & $\begin{array}{c}\text { D } \\
(\%)\end{array}$ & $\begin{array}{c}\text { EQM } \\
\left(\mathbf{L ~ h}{ }^{-1}\right)\end{array}$ \\
(I) & $\mathrm{L}_{\mathrm{c}}=\left(\frac{\mathrm{P}_{\mathrm{ei}}-\mathrm{Pst}}{\mathrm{J}}\right) 100$ & 81,61 & 0,156 \\
(II) & $\mathrm{L}_{\mathrm{c}}=\left(\frac{\mathrm{P}_{\mathrm{ei}}-\mathrm{Pst}-\mathrm{Hloc}}{\mathrm{J}}\right) 100$ & 99,99 & 0,003 \\
\hline
\end{tabular}

sendo indicada a utilização deste modelo desde que seus parâmetros sejam ajustados antecipadamente.

\section{CONClusões}

1. O modelo que desconsiderou a perda localizada de carga não estimou a vazão dos emissores com boa exatidão, pois apresentou baixo índice de concordância de Willmott.

2. O modelo que considerou a perda localizada de carga estimou a vazão dos emissores em função do comprimento do microtubo, com elevada exatidão, comprovando ser necessário considerar a perda localizada de carga para dimensionamento de sistemas de irrigação com microtubos ramificados.

\section{AgradeCIMENTos}

À Fundação de Amparo à Pesquisa do Estado de São Paulo (FAPESP), pela concessão da bolsa de estudos; ao Ministério da Ciência e Tecnologia (MCT), ao Conselho Nacional de Desenvolvimento Científico e Tecnológico (CNPq) e à Coordenação de Aperfeiçoamento de Pessoal de Nível Superior (CAPES), pelo apoio financeiro a esta pesquisa, por meio do Instituto Nacional de Ciência e Tecnologia em Engenharia da Irrigação(INCTEI).

\section{LITERATURA CITADA}

ABNT - Associação Brasileira de Normas Técnicas. Equipamentos de irrigação agrícola - Emissores e tubos emissores - Especificações e métodos de ensaio. ABNT NBR ISO 9261. São Paulo: ABNT, 2006. 17p.

Almeida, C. D. G. C.; Botrel, T. A. Determinação do diâmetro de microtubos em irrigação localizada. Revista Brasileira de Ciências Agrárias, v.5, p.413-417, 2010.

Almeida, C. D. G. C.; Botrel, T. A.; Smith, R. J. Characterization of the microtube emitters used in a novel micro-sprinkler. Irrigation Science, v.27, p.209-214, 2009.

Azevedo Netto, J. M.; Fernandez, M. F.; Araújo, R.; Ito, A. E. Manual de hidráulica. 8.ed. São Paulo: Edgard Blücher, 1998. $669 \mathrm{p}$.

Cardoso, G. G. G.; Frizzone, J. A. Perda de carga em linhas laterais de microirrigação. Revista Brasileira de Engenharia de Biossistemas, v.1, p147-162, 2007. 
Frizzone, J. A.; Vieira, A. T.; Paz, V. P. S.; Botrel, T. A. Caracterização hidráulica de um tubo gotejador. Revista Brasileira de Engenharia Agrícola e Ambiental, v.2, p.278283, 1998.

Gomes, A. W. A.; Frizzone, J. A.; Rettore Neto, O.; Miranda, J. H. Perda de carga localizada em gotejadores integrados em tubos de polietileno. Engenharia Agrícola, v.30, p.435-446, 2010.

IBGE - Instituto Brasileiro de Geografia e Estatística. Censo Agropecuário 2006: Agricultura familiar. Rio de Janeiro: IBGE, 2006. 267p.

Karmeli, D. Classification and flow regime analysis of drippers. Journal of Agricultural Engineering Research, v.22, p.165173, 1977.

Pizarro-Cabello, F. Riegos localizados de alta frecuencia (RLAF): Goteo, microaspersión, exudación. 3.ed. Madrid: MundiPrensa, 1996. 513p.

Porto, R. M. Hidráulica básica. 4.ed. São Carlos: EESC/USP, 2006. 540p.

Rettore Neto, O.; Frizzone, J. A.; Miranda, J. H.; Botrel, T. A. Perda de carga localizada em emissores não coaxiais integrados a Tubos de polietileno. Engenharia Agrícola, v.29, p.28-39, 2009.
Souza, R. O. R. M.; Botrel, T. A. Modelagem para o dimensionamento de microtubos em irrigação localizada. Revista Brasileira de Engenharia Agrícola e Ambiental, v.8, p.16-22, 2004.

Souza, R. O. R. M; Miranda, E. P.; Nascimento Neto, J. R.; Ferreira, T. T. S.; Mesquita, F. P. Irrigação localizada por gravidade em comunidades agrícolas do Ceará. Revista Ciência Agronômica, v.40, p.34-40, 2009.

Souza, R. O. R. M.; Pérez, G. F. E.; Botrel, T. A. Irrigação localizada por gravidade com microtubos. Irriga, v.11, p.266279, 2006.

Souza, W. J.; Botrel, T. A.; Almeida, A. C. S ; Correa, C. B. Modelo matemático aplicado à irrigação localizada com microtubos sob regime de escoamento turbulento. Engenharia Agrícola, v.31, p.278-289, 2011.

Willmott, C. J.; Ackleson, S. G.; Davis, R. E.; Feddema, J. J.; Klink, K. M.; Legates, D. R.; O’Donnell, J.; Rowe, C. M. Statistics for the evaluation and comparison of models. Journal of Geophysical Research, v.90, p.8995-9005, 1985.

Zitterell, D. B.; Frizzone, J. A.; Rettore Neto, O.; Ullmann, M. N. Perda de carga em microtubos e conectores utilizados em microaspersão. Engenharia Agrícola, v.29, p.591-604, 2009. 